\title{
The Effect of Time-Jitter in Equispaced Sampling Wattmeters
}

\author{
Domenico Mirri, Gaetano Iuculano, Gaetano Pasini, and Fabio Filicori
}

\begin{abstract}
This paper evaluates the effect of time-jitters in the equally spaced sampling wattmeters on the hypothesis of jitters uncorrelated with the input signals. The general case of two distinct time-jitters is considered, one common to the two channels and the other different for each one of them. The performance of the wattmeter has been evaluated by considering the asymptotic statistic parameters of the output. It has been shown that the different time-jitters introduce a bias and that both time-jitters contribute to the variance of the output. In any case, time-jitters introduce further bandwidth limitations which must be taken into account in the wattmeter accuracy evaluation. The theoretical results have been compared with simulated and experimental findings. Experimental results were obtained with a prototype in which both common and different time-jitters were separately added to the equally spaced sampling instants of the two input channels. In both cases, all the results were in good agreement with theoretical expectations.
\end{abstract}

Index Terms - Frequency response, performance analysis, sampling wattmeters, time-jitter, weighting function.

\section{INTRODUCTION}

$\mathbf{R}$ ANDOM fluctuations with respect to the nominal sampling time are commonly called timing jitter. Its effect in the amplitude and phase estimates of sampled signals [1], [2] and on the recovery of a signal from noise by averaging [3] has been studied by different authors. In a previous paper, we investigated the effect of time-jitter in digital wattmeters based on equally spaced sampling techniques on the hypothesis of a time-jitter common to the voltage and current channels and uncorrelated with the input signals [4]. In this paper, the study has been generalized by also considering the effect of different time-jitters in each channel.

Because time-jitter introduces random fluctuations in the sampling instants of the voltage and current channels, its effect can be evaluated by adding two random intervals to each nominal sampling instant, one common to the two input channels of the wattmeter and one different for each channel. Therefore, the difference between the corresponding sampling instants of the two channels is slightly random.

A criterion previously introduced by the authors [5]-[7] has been used to assess exact statements about the performance of equally spaced sampling wattmeters in the presence of time-jitters. To this end, the output quantity is expressed as a

Manuscript received January 3, 1996; revised November 30, 1998.

D. Mirri, G. Pasini, and F. Filicori are with the Facoltà di Ingegneria, University of Bologna, 40136 Bologna, Italy.

G. Iuculano is with the Facoltà di Ingegneria, University of Florence, 50139 Florence, Italy.

Publisher Item Identifier S 0018-9456(98)09795-2. function not only of the measurand, i.e., the mean cross power, and of the superimposed jitters but also of all the parameters which can influence it. Those parameters are not known $a$ priori and occur as nuisance, or incidental, quantities; the value of each of these parameters is regarded as a realization of a random variable with a priori probability distribution which must be consistent with the prior information available. By using this criterion, the uncertainty can be quantified through the asymptotic mean square error of the output of the wattmeter referred to the measurand, i.e., the mean cross power of the input signals.

In Section II, the effect of time-jitter in the equally spaced sampling wattmeter is analyzed and the output is expressed as a function of the sampling instants. In Section III, the criterion for the performance analysis is applied to this particular case. In Section IV, the expressions of the asymptotic statistic parameters of the output of the wattmeter are given on the hypothesis of jitters uncorrelated with the input signals, and the theoretical findings are compared with the simulated results. In Section V, the measurement results of a prototype in which common and different time-jitters can be separately added to the sampling instants of the input channels are compared with the theoretical ones.

\section{THE OUTPUT OF THE WATTMETER IN THE PRESENCE OF TIME-JITTER}

Let us suppose that the voltage $v(t)$ and current $i(t)$ of a single-port device are alternated signals with period $T_{1}$; therefore, the instantaneous cross power $p(t)=v(t) i(t)$ entering the one-port is periodic with the same period $T_{1}$ and can be expressed as a function of the Fourier series of $v(t)$ and $i(t)$ as follows:

$$
\begin{aligned}
p(t) & =v(t) i(t)=\sum_{\substack{m=-\infty \\
m \neq 0}}^{+\infty} \sum_{\substack{n=-\infty \\
n \neq 0}}^{+\infty} V_{m} I_{n} e^{j 2 \pi(m+n) f_{1} t} \\
& =\sum_{q=-\infty}^{+\infty} \sum_{\substack{m=-\infty \\
m \neq q \\
m \neq 0}}^{+\infty} V_{m} I_{q-m} e^{j 2 \pi q f_{1} t} \\
& =P_{0}+\sum_{\substack{q=-\infty \\
q \neq 0}}^{+\infty} P_{q} e^{j 2 \pi q f_{1} t}
\end{aligned}
$$


where $f_{1}=\left(1 / T_{1}\right), V_{-m}=V_{m}^{*}, I_{-n}=I_{n}^{*}, q=m+n$,

$$
P_{q}=\sum_{\substack{m=-\infty \\ m \neq q \\ m \neq 0}}^{+\infty} V_{m} I_{q-m}
$$

with $P_{-q}=P_{q}^{*}$ the $q$ th harmonic component of $p(t)$ (frequency-domain convolution) and

$$
P_{0}=\frac{1}{T_{1}} \int_{t-T_{1}}^{t} p(\tau) d \tau=\sum_{\substack{m=-\infty \\ m \neq 0}}^{+\infty} V_{m} I_{m}^{*}
$$

is the mean value of $p(t)$, i.e., the value of the measurand. This equation is a generalization of the Parseval theorem applied to the two periodic signals $v(t)$ and $i(t)$ and shows that the average cross power $V_{m} I_{m}^{*}$ of each $m$ th Fourier component of the input signals must be added to determine the total average power [8].

For the digital implementation of a wattmeter, the instantaneous cross power $p(t)=v(t) \cdot i(t)$ is evaluated by multiplying the sampled values of the instantaneous voltage and current. The sampling technique considered here is theoretically of an equally spaced type, i.e., any sampling instant differs from the preceding one by a constant lag $T_{c}$

$$
t_{i}=t_{i-1}+T_{c}=i T_{c}+\tau_{0}
$$

where $i$ is an integer, $T_{c}$ is the sampling interval, and $\tau_{0}$ is the shift between the initial sampling instant and the time origin of $p(t)$. In the synchronous case, $\tau_{0}$ is related to the instantaneous voltage or current through the synchronizing circuit, while in the asynchronous one $\tau_{0}$ is independent of it.

Time-jitter introduces random fluctuations with respect to the nominal sampling instants both in the voltage and current channels. For example, the noise associated with the clock generator can cause common random independent fluctuations in the sampling instants; the unpredictable temporal variations of the aperture delay of each sample and hold $(\mathrm{S} / \mathrm{H})$ of the two channels produces instead different random effects in the sampling instants of each of them. Therefore, the effects of time-jitter must be analyzed by considering separately the sampling instants of the two channels, and each of them can be determined by adding to the second term of (4) two quantities: $X_{i} T_{c}$, common to the two channels, and $X_{i}^{\prime} T_{c}$ for the voltage channel or $X_{i}^{\prime \prime} T_{c}$ for the current channel (the random variables are written in boldface). The three variables $X_{i}, X_{i}^{\prime}$, and $X_{i}^{\prime \prime}$ are the $i$ th ones of three independent sets of random continuous variables with null mean value. We assume that the two variables $X_{i}^{\prime}$ and $X_{i}^{\prime \prime}$ have the same distribution with a common density function $f_{1}(x)$ and their characteristic function is defined as follows:

$$
\begin{aligned}
\Phi_{1}\left(f T_{c}\right) & =M\left\{e^{j 2 \pi f T_{c} X_{i}^{\prime}}\right\}=M\left\{e^{j 2 \pi f T_{c} X_{i}^{\prime \prime}}\right\} \\
& =\int_{-\infty}^{+\infty} e^{j 2 \pi f T_{c} x} f_{1}(x) d x
\end{aligned}
$$

where $\Phi_{1}\left(-f T_{c}\right)=\Phi_{1}^{*}\left(f T_{c}\right)$ and $\Phi_{1}(0)=1$. Further, we indicate by $\Phi\left(f T_{c}\right)$ the characteristic function of the variable $X_{i}$

$$
\Phi\left(f T_{c}\right)=M\left\{e^{j 2 \pi f T_{c} X_{i}}\right\}=\int_{-\infty}^{+\infty} f(x) e^{j 2 \pi f T_{c} x} d x
$$

with $\Phi\left(-f T_{c}\right)=\Phi^{*}\left(f T_{c}\right)$ and $\Phi(0)=1$.

The generic discrete output of the sampling wattmeter, marked with an integer $k$, is an estimate of $P_{0}$ [see (3)] and can be deduced by forming a weighted average of the last $N$ successive values of the instantaneous power [see (1)]

$$
\begin{aligned}
\hat{\boldsymbol{P}}_{k} & =\sum_{i=0}^{N-1} a_{i} v\left(\boldsymbol{t}_{k-i}^{\prime}\right) i\left(\boldsymbol{t}_{k-i}^{\prime \prime}\right) \\
& =\sum_{\substack{m=-\infty \\
m \neq 0}}^{+\infty} \sum_{\substack{n=-\infty \\
n \neq 0}}^{+\infty} V_{m} I_{n} \sum_{i=0}^{N-1} a_{i} e^{j 2 \pi f_{1}\left(m \boldsymbol{t}_{k-i}^{\prime}+n \boldsymbol{t}_{k-i}^{\prime \prime}\right)} \\
& =\sum_{q=-\infty}^{+\infty} \sum_{\substack{m=-\infty \\
m \neq 0 \\
m \neq q}}^{+\infty} V_{m} I_{q-m} \sum_{i=0}^{N-1} a_{i} e^{j 2 \pi f_{1}\left[m \boldsymbol{t}_{k-i}^{\prime}+(q-m) \boldsymbol{t}_{k-i}^{\prime \prime}\right]}
\end{aligned}
$$

where the random variables $t_{k-i}^{\prime}$ and $t_{k-i}^{\prime \prime}$ are the sampling instants of the voltage and current channels

$$
\begin{aligned}
& t_{k-i}^{\prime}=\tau_{0}+\left(k-i+X_{k-i}+X_{k-i}^{\prime}\right) T_{c} \\
& t_{k-i}^{\prime \prime}=\tau_{0}+\left(k-i+X_{k-i}+X_{k-i}^{\prime \prime}\right) T_{c} .
\end{aligned}
$$

Obviously, $t_{k-i}^{\prime}$ and $t_{k-i}^{\prime \prime}$ are mutually interdependent due to the common component $X_{k-i}$; further, their mean values coincide with (4) if $i$ is substituted with $k-i$.

By taking into account (8) and (9), (7) can be rewritten as follows:

$$
\begin{aligned}
\hat{P}_{k}= & \sum_{q=-\infty}^{+\infty} \sum_{\substack{m=-\infty \\
m \neq 0 \\
m \neq q}}^{+\infty} V_{m} I_{q-m} e^{j 2 \pi q f_{1}\left(\tau_{0}+k T_{c}\right)} \\
& \cdot \sum_{i=0}^{N-1} a_{i} e^{-j 2 \pi q f_{1} T_{c}} e^{j 2 \pi f_{1} T_{c}\left(q \boldsymbol{X}_{k-i}+m \boldsymbol{X}_{k-i}^{\prime}+(q-m) \boldsymbol{X}_{k-i}^{\prime \prime}\right)}
\end{aligned}
$$

which defines the influence of both common and different time-jitters on any measured value $\hat{\boldsymbol{P}}_{k}$; time-jitters are represented by the random variables $X_{k-i}, X_{k-i}^{\prime}$, and $X_{k-i}^{\prime \prime}$. When time-jitters are missing [(10) with $\boldsymbol{X}_{k-i}=\boldsymbol{X}_{k-i}^{\prime}=$ $\left.\boldsymbol{X}_{k-i}^{\prime \prime}=0\right]$, the discrete output of the equally spaced sampling wattmeter can be easily deduced by recalling (2) [4]; in particular, by imposing $a_{i}=1 / N$, the synchronous sampling strategy can be studied [5], [6], [9], in which strategy the $N$ sampling instants are taken in $r$ periods $T_{1}$

$$
f_{1} T_{c}=\frac{r}{N}
$$

with $r$ a positive integer having no common submultiple with $N$. 


\section{CRITERION FOR THE PERFORMANCE ANALYSIS}

The output quantity $\hat{P}_{k}=f\left(\tau_{0}, \underline{X}_{k}, \underline{X}_{k}^{\prime}, \underline{X}_{k}^{\prime \prime}\right)$ in (10) depends not only on the time-jitters through the vectors $\underline{X}_{k}$, $\underline{X}_{k}^{\prime}, \underline{X}_{k}^{\prime \prime}$ ) of the random variables $X_{k-i}, X_{k-i}^{\prime}, X_{k-i}^{\prime \prime}$ (with $i=0 \cdots N-1)$, but also on two parameters $\left(k, \tau_{0}\right)$ which are unknown a priori. Although these parameters are essential to evaluate $\hat{\boldsymbol{P}}_{k}$, they are a nuisance in the formulation of exact statements about the performance. The labeling mark $k$ identifies a generic discrete output of the instrument; therefore, the integer $k$ is random in nature because it can be considered randomly picked up from a sequence of $2 h+1$ successive potential measurement occasions $(-h \leq k \leq+h)$, each of which has an equal chance of being selected. In the asynchronous case, the shift $\tau_{0}$ is strictly related to the turnon instant of the instrument. Instead, in the synchronous operation, the shift $\tau_{0}$ depends on the synchronizing circuit used and, possibly, also on the waveform of the signal at its input. In both cases, the value of $\tau_{0}$ is not known a priori and one is led to interpret any value of $\tau_{0}$ as an outcome of a random variable with a continuous set of values uniformly distributed in some generic time interval $-T / 2 \leq \tau_{0} \leq+T / 2$ ( $T$ being unknown) which ideally encompasses all the possible circuital implementations. Therefore, we introduce $k$ as a discrete random variable uniformly distributed in the interval $(-h,+h)$ and $\tau_{0}$ as a continuous random variable uniformly distributed in the time interval $(-T / 2,+T / 2)$. Obviously, the variability of the output in the synchronous sampling strategy without time-jitter is due uniquely to the hypothesized variability of $\tau_{0}$ [5], [6]. The statements of $h$ and $T$ are subjective guesses based on available known properties of the measuring instrument.

On the basis of the proposed criterion, the output quantity $\hat{\boldsymbol{P}}_{\boldsymbol{k}}=f\left(\tau_{0}, \underline{\boldsymbol{X}}_{k}, \underline{\boldsymbol{X}}_{k}^{\prime}, \underline{\boldsymbol{X}}_{k}^{\prime \prime}\right)$ becomes a function of the random variables $k, \tau_{0}$, and of the vectors $\left.\underline{X}_{k}, \underline{X}_{k}^{\prime}, \underline{X}_{k}^{\prime \prime}\right)$ of the random variables $\underline{\boldsymbol{X}}_{k-i}, \underline{\boldsymbol{X}}_{k-i}^{\prime}, \underline{\boldsymbol{X}}_{k-i}^{\prime \prime}$ ) (with $i=0, \cdots, N-1$ ) used to obtain the output corresponding to $\boldsymbol{k}$. An appropriate characterization of the output uncertainty can be obtained by evaluating the statistical parameters of $\hat{\boldsymbol{P}}_{k}$, i.e., the mean value $M\left\{\hat{\boldsymbol{P}}_{k}\right\}$ and the mean square error $M\left\{\left(\hat{\boldsymbol{P}}_{\boldsymbol{k}}-P_{0}\right)^{2}\right\}$. In order to incorporate all the a priori chances into the instrument's performance, in the asynchronous case the number of the measurement occasions, i.e., $2 h+1$, must be sufficiently large and theoretically tend to infinite; in the synchronous one, it is the excursion of the initial shift $\tau_{0}$, i.e., $T$, which must tend to infinite [6]. Therefore, in any case, we consider the asymptotic statistical parameters, i.e., the asymptotic mean

$$
\underset{\rightarrow}{\rightarrow}\left\{\hat{\boldsymbol{P}}_{\boldsymbol{k}}\right\}=\lim _{\substack{T \rightarrow \infty \\ \text { or } h \rightarrow \infty}} M\left\{\hat{\boldsymbol{P}}_{\boldsymbol{k}}\right\}
$$

and the asymptotic mean square error

$$
\begin{aligned}
\stackrel{E}{\rightarrow} & =\underset{\rightarrow}{M}\left\{\left(\hat{\boldsymbol{P}}_{\boldsymbol{k}}-P_{0}\right)^{2}\right\} \\
& =\underset{\operatorname{var}}{\operatorname{va}}\left\{\hat{\boldsymbol{P}}_{\boldsymbol{k}}\right\}+\left(P_{0}-\underset{\rightarrow}{M}\left\{\hat{\boldsymbol{P}}_{\boldsymbol{k}}\right\}\right)^{2} \\
& =\underset{\rightarrow}{M}\left\{\hat{\boldsymbol{P}}_{\boldsymbol{k}}^{2}\right\}-2 P_{0} \underset{\rightarrow}{M}\left\{\hat{\boldsymbol{P}}_{\boldsymbol{k}}\right\}+P_{0}^{2}
\end{aligned}
$$

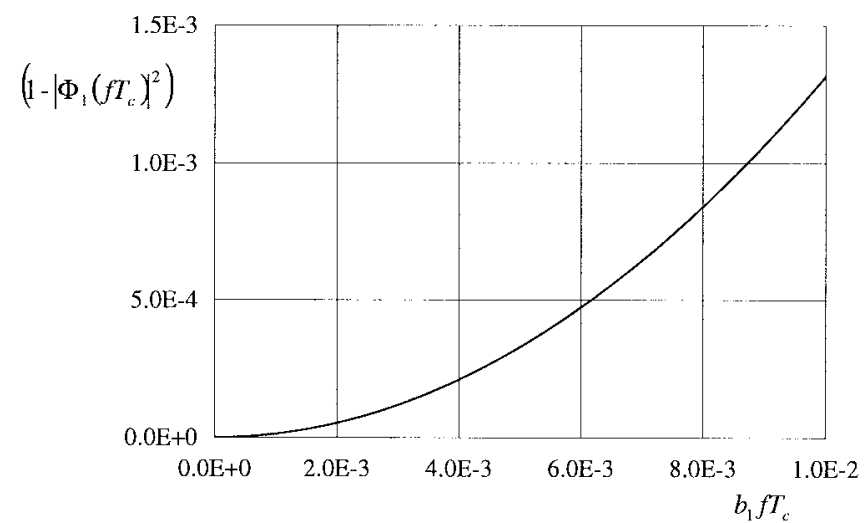

Fig. 1. Plot of the weighting function $\left(1-\left|\Phi_{1}\left(f T_{c}\right)\right|^{2}\right)$ in (15) in the presence of different jitters in two channels with normal or uniform distribution.

where

$$
\underset{\operatorname{var}}{\rightarrow}\left\{\hat{\boldsymbol{P}}_{\boldsymbol{k}}\right\}=\underset{\rightarrow}{M}\left\{\left(\hat{\boldsymbol{P}}_{\boldsymbol{k}}-\underset{\rightarrow}{M}\left\{\hat{\boldsymbol{P}}_{\boldsymbol{k}}\right\}\right)^{2}\right\}=\underset{\rightarrow}{M}\left\{\hat{\boldsymbol{P}}_{\boldsymbol{k}}^{2}\right\}-\underset{\rightarrow}{M^{2}}\left\{\hat{\boldsymbol{P}}_{\boldsymbol{k}}\right\}
$$

is the asymptotic variance while $P_{0}-M\left\{\hat{\boldsymbol{P}}_{\boldsymbol{k}}\right\}$ is the asymptotic bias.

\section{TheORETICAL AND Simulation RESUlts}

By assuming that the correct scaling condition $\sum_{i=0}^{N-1} a_{i}=1$ is satisfied [4], it can be shown that the asymptotic mean output value can be expressed as follows [see (A6) in the Appendix]:

$$
\underset{\rightarrow}{M}\left\{\hat{\boldsymbol{P}}_{\boldsymbol{k}}\right\}=\sum_{\substack{m=-\infty \\ m \neq 0}}^{+\infty} V_{m} I_{m}^{*}\left|\Phi_{1}\left(m f_{1} T_{c}\right)\right|^{2}
$$

where $\Phi_{1}\left(f T_{c}\right)$ is the characteristic function common to the two random variables $\boldsymbol{X}_{i}^{\prime}$ and $\boldsymbol{X}_{i}^{\prime \prime}$ which characterize the different time-jitters of the two channels (5). From (14), we deduce that the presence of separate time-jitters in the two channels determines an asymptotic bias which depends on their common characteristic function

$$
\underset{\rightarrow}{\operatorname{Bias}}=P_{0}-\underset{\rightarrow}{M}\left\{\hat{\boldsymbol{P}}_{\boldsymbol{k}}\right\}=\sum_{\substack{m=-\infty \\ m \neq 0}}^{+\infty} V_{m} I_{m}^{*}\left(1-\left|\Phi_{1}\left(m f_{1} T_{c}\right)\right|^{2}\right) .
$$

In particular, the characteristic function results

$$
\Phi_{1}\left(f T_{c}\right)=\operatorname{sinc}\left(2 b_{1} f T_{c}\right)
$$

in the case of a uniform distribution in the interval $\pm b_{1} T_{c}$ or

$$
\Phi_{1}\left(f T_{c}\right)=e^{-2\left(\pi \sigma_{1} T_{c}\right)^{2}}
$$

in the case of a normal one with mean zero and standard deviation $\sigma_{1}$. To compare the effects of these two random distributions, we can assume for both the same variance, i.e., $\sigma_{1}^{2}=b_{1}^{2} / 3$. In Fig. 1 , the two weights $\left(1-\operatorname{sinc}^{2}\left(2 b_{1} f T_{c}\right)\right)$ and $\left(1-e^{-4\left(\pi \sigma_{1} f T_{c}\right)^{2}}\right)$ are plotted as functions of $b_{1} f T_{c}$ in the range of the weights from zero to $1.5 \cdot 10^{-3}$. It should be noted that, in this range, they practically coincide. It is interesting to observe that, according to this plot, the separate time-jitters in the two channels actually introduce a bandwidth limitation in 
TABLE I

Maximum Frequency $f_{\max }$ Corresponding to Two Prefixed UpPER Limits $\left(10^{-3}, 10^{-4}\right)$ OF THE TheORETICAL Asymptotic Bías When $b_{1}=0.01$ FOR SinUSOIdal InPUT Signals With $P_{0}=1 \overrightarrow{\mathrm{W}}$. The Difference $\Delta \underset{\text { Bias }}{\rightarrow}$ with Respect to the Simulated Value $\Delta$ Bías is Given for Four Different Values of the Phase $\varphi$

\begin{tabular}{ccccc}
\hline$\underset{\operatorname{Bias}}{\longrightarrow}(\mathrm{W})$ & $f_{\max }(\mathrm{kHz})$ & $P_{0}(\mathrm{~W})$ & $\varphi$ & $\underset{\Delta \text { Bias }}{\longrightarrow}$ \\
\hline $1 \cdot 10^{-3}$ & 43.55 & 1.0 & 0 & $0.46 \cdot 10^{-4}$ \\
$1 \cdot 10^{-3}$ & 43.55 & 1.0 & 30 & $-0.13 \cdot 10^{-4}$ \\
$1 \cdot 10^{-3}$ & 43.55 & 1.0 & 45 & $-0.41 \cdot 10^{-4}$ \\
$1 \cdot 10^{-3}$ & 43.55 & 1.0 & 60 & $-0.75 \cdot 10^{-4}$ \\
$1 \cdot 10^{-4}$ & 13.75 & 1.0 & 0 & $0.067 \cdot 10^{-4}$ \\
$1 \cdot 10^{-4}$ & 13.75 & 1.0 & 30 & $-0.16 \cdot 10^{-4}$ \\
$1 \cdot 10^{-4}$ & 13.75 & 1.0 & 45 & $0.16 \cdot 10^{-4}$ \\
$1 \cdot 10^{-4}$ & 13.75 & 1.0 & 60 & $0.23 \cdot 10^{-4}$ \\
\hline
\end{tabular}

the input signals, if we assume that the measurement bias due to time-jitter should not exceed the contributions due to other causes of error in the wattmeter. In fact, once the parameter $\pm b_{1} T_{c}$ has been fixed, the bandwidth of the instrument due to the time-jitter can be deduced by imposing a maximum value of the weight in (15).

In order to validate (15), a synchronous wattmeter with a sampling interval $T_{c}=200 \mu \mathrm{s}$ was simulated. By assuming $f_{1}=50 \mathrm{~Hz}, r=1$, and consequently $N=10^{3}$ according to (11), the asymptotic bias in the absence of time-jitter is null and only the spectral components' multiple integer of $N f_{1}=50 \mathrm{kHz}$ contributes to the estimated mean value (see $[4$, eq. (8)]). In the presence of different time-jitters in the two channels, the average cross power $V_{m} I_{m}^{*}$ of each $m$ th Fourier component of the input signals, weighted by the squared modulus $\left|\Phi_{1}\right|^{2}$ of the common characteristic function, gives a contribution to the asymptotic mean value (14), leading to the corresponding bias according to (15). On the hypothesis of a uniform distribution of the sampling instants in the interval $\pm b_{1} T_{c}$ with $\pm b_{1}= \pm 0.01$, the upper limit of the frequency $\left(f_{\max }\right)$ of the input signals for two given upper limits of the asymptotic bias $\left(10^{-3}\right.$ and $\left.10^{-4}\right)$ was theoretically deduced from (15). By assuming a unique spectral component at this frequency $f_{\max }$ and a unit mean power, the asymptotic bias was estimated for four phase shifts $\varphi$ between the two input signals. The results are reported in Table I, where Bias $\rightarrow$ is the theoretical asymptotic bias, $\underset{\text { Bias }}{\rightarrow}$ is the estimated one, and

$$
\underset{\sim}{\operatorname{Bias}}=\underset{\rightarrow}{\operatorname{Bias}}-\underset{\text { Bias }}{\rightarrow}
$$

is their difference. The experimental results of the simulated instrument are in very good agreement with the theoretical findings.

On the hypothesis of a rectangular filtering window $\left[a_{i}=\right.$ $1 / N$ in (10)], the asymptotic mean squared error (13) in the presence of different and common time-jitters can be expressed as (18), shown at the bottom of the page [see (A6) and (A13) in the Appendix], where $\Phi_{1}\left(f T_{c}\right)$ and $\Phi\left(f T_{c}\right)$ are the characteristic functions of the random variables which characterize the different and the common time-jitters of the two channels (5) and (6), respectively, while $H\left(f T_{c}\right)$ is the frequency response of the digital filter [4]

$$
H\left(f T_{c}\right)=e^{-j \pi f T_{c}(N-1)} \frac{\operatorname{sinc}\left(N f T_{c}\right)}{\operatorname{sinc}\left(f T_{c}\right)} .
$$

According to (18), it can be easily shown that, for $N$ tending to infinite, the residual asymptotic mean square error coincides with the squared bias

$$
\lim _{N \rightarrow \infty} E^{2}=\left[\sum_{m=-\infty}^{+\infty} V_{m} I_{m}^{*}\left(1-\left|\Phi_{1}\left(m f_{1} T_{c}\right)\right|^{2}\right)\right]^{2}=\text { Bias }^{2}
$$

due to the fact that (see $[4$, eq. (8)])

$$
\lim _{n \rightarrow \infty}\left|H\left(q f_{1} T_{c}\right)\right|^{2}=\left\{\begin{array}{ll}
0, & \text { for } q \neq 0 \\
1, & \text { for } q=0
\end{array} .\right.
$$

In the case of two sinusoidal input signals at frequency $f_{1}$, it can be shown that the asymptotic variance, when the effect of the common time-jitter is negligible, can be expressed as follows:

$$
\begin{aligned}
\stackrel{2}{\rightarrow}=2\left|V_{1} I_{1}^{*}\right|^{2} & \left\{\frac{2}{N}\left(1-\left|\Phi_{1}\left(f_{1} T_{c}\right)\right|^{4}\right)+\left|\Phi_{1}\left(f_{1} T_{c}\right)\right|^{4}\left|H\left(2 f_{1} T_{c}\right)\right|^{2}\right\} \\
& +\frac{P_{0}^{2}}{2}\left\{\frac{1}{N}\left[\left.\Phi_{1}\left(2 f_{1} T_{c}\right)\right|^{2}-\left|\Phi_{1}\left(f_{1} T_{c}\right)\right|^{4}\right]\right\} \cdot(22)
\end{aligned}
$$

The normalized quantity

$$
\underset{\rightarrow}{\sigma_{N}^{2}}=\underset{\rightarrow}{\sigma^{2}} / 2\left|V_{m} I_{m}^{*}\right|^{2}
$$

$$
\begin{aligned}
\stackrel{2}{E}=\underset{\rightarrow}{M}\left\{\left(\hat{P}_{k}-P_{0}\right)^{2}\right\}= & \frac{1}{N} \sum_{q=-\infty}^{+\infty} \sum_{\substack{m=-\infty \\
m \neq q \\
m \neq 0}}^{+\infty} \sum_{\substack{m^{\prime}=-\infty \\
m \neq q \\
m \neq 0}}^{+\infty} V_{m} I_{q-m} V_{m^{\prime}} I_{-q-m^{\prime}}\left|\Phi_{1}\left(\left(m+m^{\prime}\right) f_{1} T_{c}\right)\right|^{2} \\
& +\sum_{q=-\infty}^{+\infty}\left|\sum_{\substack{m=-\infty \\
m \neq q \\
m \neq 0}}^{+\infty} V_{m} \Phi_{1}\left(m f_{1} T_{c}\right) I_{q-m} \Phi_{1}\left((q-m) f_{1} T_{c}\right)\right|\left|\Phi\left(q f_{1} T_{c}\right)\right|^{2}\left[\left|H\left(q f_{1} T_{c}\right)\right|^{2}-\frac{1}{N}\right] \\
& -2 P_{0} \sum_{\substack{m=-\infty \\
m \neq 0}}^{+\infty} V_{m} I_{m}^{*}\left|\Phi_{1}\left(m f_{1} T_{c}\right)\right|^{2}+P_{0}^{2}
\end{aligned}
$$




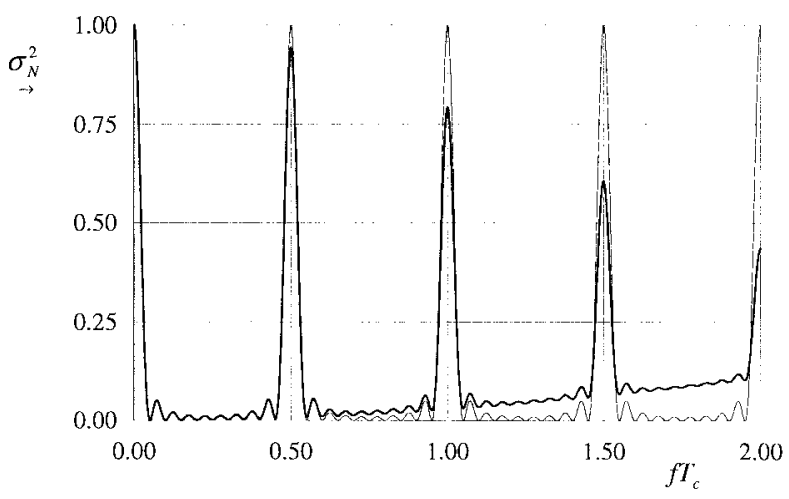

Fig. 2. Plot of the normalized variance $\sigma_{N}^{2}=\underset{\sigma^{2}}{\rightarrow} / 2\left|V_{m} I_{m}^{*}\right|^{2}$ (22) as a function of $f T_{c}$ in the presence (thick line) or absence (thin line) of time-jitters different for the two channels, on the hypothesis of $\pm b_{1}= \pm 0.01, N=10$ and input signals in phase.

TABLE II

Comparison $\tilde{\Delta \sigma}$ BetweEn THE THEORETICAL $\sigma$ AND Simulated $\tilde{\sigma} \vec{A}$ Asymptotic Standard ERror IN THE Presence of ONLY Different Time-JitTERS AND Under THE Same Operating Conditions Considered for TABle I

\begin{tabular}{|c|c|c|c|c|}
\hline$\underset{\sigma}{\sigma}(\mathrm{W})$ & $f_{\max }(\mathrm{kHz})$ & $P_{0}(\mathrm{~W})$ & $\varphi$ & $\tilde{\Delta \sigma}$ \\
\hline $0.999 \cdot 10^{-3}$ & 43.55 & 1.0 & 0 & $-0.068 \cdot 10^{-4}$ \\
\hline $1.41 \cdot 10^{-3}$ & 43.55 & 1.0 & 30 & $0.30 \cdot 10^{-4}$ \\
\hline $2.00 \cdot 10^{-3}$ & 43.55 & 1.0 & 45 & $-0.19 \cdot 10^{-4}$ \\
\hline $3.16 \cdot 10^{-3}$ & 43.55 & 1.0 & 60 & $0.084 \cdot 10^{-4}$ \\
\hline $0.315 \cdot 10^{-3}$ & 13.75 & 1.0 & 0 & $-0.17 \cdot 10^{-4}$ \\
\hline $0.446 \cdot 10^{-3}$ & 13.75 & 1.0 & 30 & $0.081 \cdot 10^{-4}$ \\
\hline $0.631 \cdot 10^{-3}$ & 13.75 & 1.0 & 45 & $-0.12 \cdot 10^{-4}$ \\
\hline $0.998 \cdot 10^{-3}$ & 13.75 & 1.0 & 60 & $-0.24 \cdot 10^{-4}$ \\
\hline
\end{tabular}

for input signals in phase is plotted in Fig. 2 as a function of $f T_{c}$, in the presence (thick line) and absence (thin line) of different time-jitters, on the hypothesis of sampling instants uniformly distributed in the jitter interval $\pm b_{1} T_{c}$ with $\pm b_{1}=$ $\pm 0.01, N=10$. These values of $\pm b_{1}$ and $N$ were assumed in order to highlight the effect of time-jitter; in particular, the variance at the frequencies corresponding to the null values of the spectral response of the filter [see (19)] becomes increasingly greater with frequency, while those corresponding to the peak-amplitude values are instead attenuated.

The asymptotic standard error has been computed, according to (22), under the same operating conditions considered for Table I, and Table II reports the comparison with the corresponding simulated values $(\tilde{\Delta} \sigma=\sigma-\tilde{\sigma})$.

When the effect of both the random variables $\boldsymbol{X}^{\prime}$ and $X^{\prime \prime}$ due to the different time-jitters is negligible, we can assume $\Phi_{1}\left(f T_{c}\right)=1$. In this case, we obviously obtain the same results already outlined in [4], i.e., the asymptotic mean of the output coincides with the mean value of $p(t)$

$$
\underset{\rightarrow}{M}\left\{\hat{P}_{k}\right\}=P_{0}
$$

and the asymptotic mean squared error coincides with the

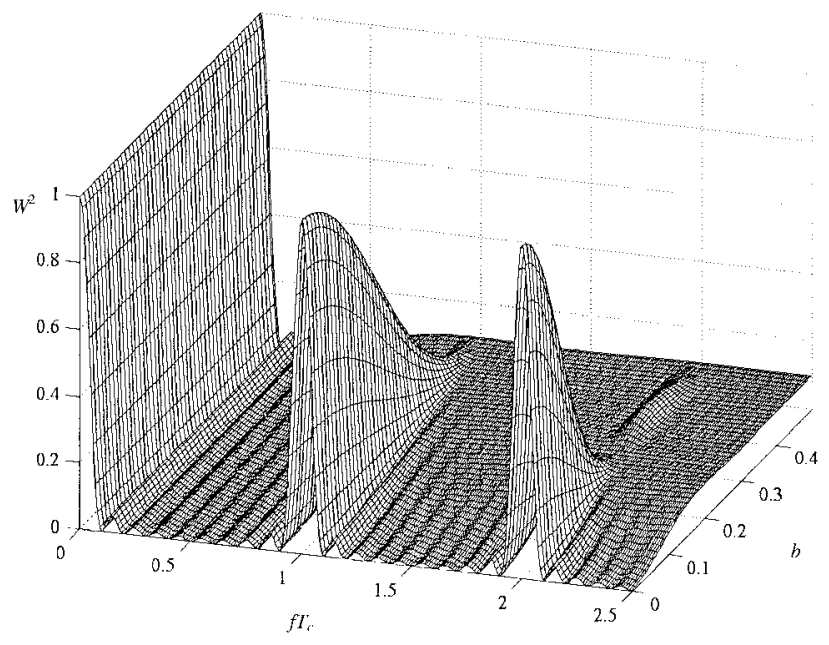

Fig. 3. Shape of the weight $W^{2}(25)$ as a function both of $f T_{c}$ and $b\left(2 b T_{c}\right.$ is the time interval in which each sampling instant is uniformly distributed).

asymptotic variance [see (18)]

$$
\stackrel{E^{2}}{\rightarrow}=\sigma^{2}=2 \sum_{q=1}^{\infty}\left|P_{q}\right|^{2} W^{2}\left(q f_{1} T_{c}\right)
$$

where $[4$, eq. (16)]

$$
W^{2}\left(f T_{c}\right)=\frac{1}{N}\left(1-\left|\Phi\left(f T_{c}\right)\right|^{2}\right)+\left|\Phi\left(f T_{c}\right)\right|^{2} \frac{\operatorname{sinc}^{2}\left(N f T_{c}\right)}{\operatorname{sinc}^{2}\left(f T_{c}\right)}
$$

with $W^{2}(0)=1$ is a function which weights the contribution of each spectral component of the instantaneous power to the asymptotic variance. Finally, when the effect of all the random variables is negligible, we can assume also $\Phi\left(f T_{c}\right)=1$ and the weighting function (24) becomes coincident with the square of the modulus of the frequency response of the digital filter used, as it is well known [4].

It can be noted that, in the special case of $b=0.5,(23)-(25)$ lead to the results found for a random sampling strategy proposed by the authors [10]. In this case, in fact, as it can be seen from the plot in Fig. 3, a time-jitter with uniform distribution with $b=0.5$ gives better frequency-independent performance in the high frequency range; therefore, this special type of "jitter" actually corresponds to a convenient sampling strategy.

\section{EXPERIMENTAL RESULTS}

A sampling wattmeter with $T_{c}=50 \mu$ s has been realized to experimentally evaluate the effects of time-jitters. To this end, the sampling time generator of the wattmeter has been modified in order to sum separately common or different timejitters to the equally spaced sampling instants of the voltage and current channels; the selected random distribution of the sampling instants has been the uniform one. By imposing $N=32$ and by considering $2^{10}$ successive measurements, the effects of common and different time-jitters have been evaluated (on the hypothesis of input signals in phase) at the frequencies of the input signals corresponding to null values of the digital filter [see (19)] in the range $5.0-9.7 \mathrm{kHz}$ of the input 
TABLE III

Normalized Theoretical Asymptotic Bias $\operatorname{Bias}_{N}=\operatorname{Bias}_{\rightarrow} / P_{0}$ (15) And StANDARd ERror $\sigma_{N}=\underline{\sigma}(22)$ AND Their DifFERENCEs $\Delta \operatorname{Bias}_{N}^{\prime}$, $\Delta \sigma_{N}^{\prime}$ With Respect to the ExPERIMENTAL Values, When Only DIFFERENT TIME-JitTERS UNIFORMLY DistRIBUTED IN THE INTERVAL $\pm 0.094 T_{c}$ ARE Present, at FreQuencies of THE Sinusoidal INPUT Signals Corresponding to Null Values of the Digital Filter (19)

\begin{tabular}{ccccc}
\hline$f(\mathrm{~Hz})$ & $\operatorname{Bias}_{N}(\mathrm{~W})$ & $\Delta \operatorname{Bias}_{N}^{\prime}(\mathrm{W})$ & $\underset{N}{\longrightarrow}(\mathrm{W})$ & $\Delta \sigma_{N}^{\prime}(\mathrm{W})$ \\
\hline 5000 & 0.00795 & 0.0026 & 0.0224 & 0.0069 \\
5312.5 & 0.0898 & 0.0031 & 0.0238 & 0.0086 \\
5625 & 0.0101 & 0.0019 & 0.0252 & 0.0072 \\
5937.5 & 0.0112 & 0.0025 & 0.0266 & 0.0091 \\
6250 & 0.0124 & 0.0018 & 0.0280 & 0.0077 \\
6562.5 & 0.0137 & 0.0028 & 0.0294 & 0.0074 \\
6875 & 0.0150 & 0.0032 & 0.0308 & 0.0098 \\
7187.5 & 0.0164 & 0.0025 & 0.0322 & 0.0086 \\
7500 & 0.0178 & 0.0024 & 0.0336 & 0.0075 \\
7812.5 & 0.0193 & 0.0023 & 0.0351 & 0.0096 \\
8125 & 0.0209 & 0.0018 & 0.0365 & 0.0064 \\
8437.5 & 0.0225 & 0.0027 & 0.0379 & 0.0095 \\
8750 & 0.0242 & 0.0031 & 0.0393 & 0.0092 \\
9062.5 & 0.0259 & 0.0037 & 0.0407 & 0.0083 \\
9375 & 0.0277 & 0.0043 & 0.0421 & 0.0073 \\
9687.5 & 0.0296 & 0.0044 & 0.0436 & 0.0069 \\
\hline
\end{tabular}

signals. Table III reports, in the presence of only different timejitters uniformly distributed in the interval $\pm b_{1} T_{c}$ with $b_{1}=$ 0.094 , the theoretical values of the normalized asymptotic bias $\operatorname{Bias}_{N}=\operatorname{Bias} / P_{0}$ (15) and standard error $\sigma_{N}=$ $\underset{\rightarrow}{\sigma} / \sqrt{2}\left|V_{m} I_{m}^{*}\right|(22)$ and their differences $\Delta \operatorname{Bias}_{N}^{\prime}, \Delta \sigma_{N}^{\prime}$ with

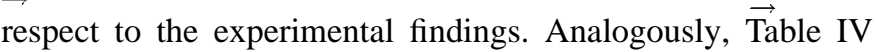
reports, in the presence of only a common time-jitter uniformly distributed in the same interval, the theoretical values of the normalized standard error $\underset{N}{\sigma_{N}}=\underset{\rightarrow}{\sigma} / \sqrt{2}\left|P_{q}\right|$ (24), together the experimental ones $\sigma_{N}^{\prime \prime}$ and their difference $\Delta \sigma_{N}^{\prime \prime}$ under the same operating conditions of Table III. Bearing in mind that we are considering causes of error, the experimental results are in good agreement with the theoretical findings.

\section{CONCLUSIONS}

This paper evaluates the effect of time-jitter on the equally spaced sampling wattmeter. Time-jitter introduces random fluctuations with respect to the nominal sampling instants both in the voltage and current channels; it can be caused by elements which are common to the two channels, or different. The latter makes the sampling instants of the two channels slightly different.

The effect of time-jitter can be evaluated by superimposing two random intervals on the nominal sampling instants, one common to both input channels and the other separate for each channel. We postulate that the two random variables, which take into account the separated time-jitter, are identically distributed and that time-jitters are uncorrelated to the input signals.

According to a criterion previously introduced by the authors, the performance of the instrument has been evaluated by considering the statistical properties of the output, i.e., the
TABLE IV

Theoretical Values of the Normalized Standard ERror $\underset{\sim}{\sigma_{N}}=\underline{\sigma} / \sqrt{2}\left|P_{q}\right|(24)$, EXPERIMENTAL ONES $\sigma_{N}^{\prime \prime}$, AND THEIR

DifFerence $\Delta \sigma_{N}^{\prime \prime}$, When Only Common Time-JitTers ARE Present, Under the Same Operating Conditions of Table III

\begin{tabular}{cccc}
\hline$f(\mathrm{~Hz})$ & $\sigma_{N}(\mathrm{~W})$ & $\sigma_{N}^{\prime \prime}(\mathrm{W})$ & $\Delta \sigma_{N}^{\prime \prime}(\mathrm{W})$ \\
\hline 5000 & 0.0314 & 0.0378 & 0.0064 \\
5312.5 & 0.0333 & 0.0368 & 0.0035 \\
5625 & 0.0352 & 0.0386 & 0.0033 \\
5937.5 & 0.0372 & 0.0402 & 0.0030 \\
6250 & 0.0391 & 0.0402 & 0.0011 \\
6562.5 & 0.0410 & 0.0426 & 0.0016 \\
6875 & 0.0429 & 0.0461 & 0.0032 \\
7187.5 & 0.0448 & 0.0480 & 0.0032 \\
7500 & 0.0467 & 0.0459 & -0.00077 \\
7812.5 & 0.0486 & 0.0511 & 0.0025 \\
8125 & 0.0465 & 0.0526 & 0.0021 \\
8437.5 & 0.0523 & 0.0547 & 0.0024 \\
8750 & 0.0542 & 0.0568 & 0.0026 \\
9062.5 & 0.0561 & 0.0563 & 0.00024 \\
9375 & 0.0579 & 0.0591 & 0.0012 \\
9687.5 & 0.0597 & 0.0607 & 0.00097 \\
\hline
\end{tabular}

asymptotic mean and the asymptotic mean square error. It has been shown that an asymptotic bias appears when different time-jitters for the two channels are present (a typical example is the time-jitters separately introduced by each $\mathrm{S} / \mathrm{H}$ ); this bias can be deduced by weighting the mean cross power of the individual Fourier components of the input signals with a function that is the complement to the squared modulus of the common characteristic function of the time-jitters. In order to obtain a bias comparable with the accuracy of the instrument, the maximum frequency of the input signal must therefore be limited. The simulated and experimental results obtained with a prototype wattmeter are in very good agreement with the theoretical findings.

The asymptotic mean square error is influenced both by common and separate time-jitters. The general expression of the asymptotic mean squared error has been theoretically deduced, and a simplified expression of the asymptotic variance has been deduced in the presence of only different time-jitters for the two channels on the hypothesis of two sinusoidal input signals. When the effect of the different time-jitters is negligible, the asymptotic variance can be obtained by weighting each spectral component of the instantaneous power, excluding the dc one, with a convenient function. Due to these effects, a further bandwidth limitation is introduced. Both simulated and experimental results, obtained with a prototype wattmeter, have confirmed the validity of the theoretical findings for each of the two types of time-jitters.

\section{APPENDIX}

For the evaluation of the statistical mean of $\hat{P}_{k}$, we must consider the simultaneous contributions of the independent random variables $\tau_{0}$ and $\boldsymbol{k}$ and of the independent random vectors $\underline{X}_{\boldsymbol{k}}, \underline{X}_{\boldsymbol{k}}^{\prime}$, and $\underline{X}_{\boldsymbol{k}}^{\prime \prime}$. The components of each random vector are mutually independent and identically distributed with a common probability density which is independent from 
$\boldsymbol{k}$. By introducing the random variables $\tau_{0}$ and $k$ into (10), we deduce

$$
\begin{aligned}
M\left\{\hat{\boldsymbol{P}}_{\boldsymbol{k}}\right\}= & \sum_{q=-\infty}^{+\infty} \sum_{\substack{m=-\infty \\
m \neq q \\
m \neq 0}}^{+\infty} V_{m} I_{q-m} M\left\{e^{j 2 \pi q f_{1} \tau_{0}}\right\} \\
& \cdot M\left\{e^{j 2 \pi q f_{1} \boldsymbol{k} T_{c}}\right\} \sum_{i=0}^{N-1} a_{i} e^{-j 2 \pi q f_{1} T_{c}} \\
& \cdot M\left\{e^{j 2 \pi q f_{1} \boldsymbol{X}_{\boldsymbol{k}-i} T_{c}}\right\} M\left\{e^{j 2 \pi f_{1} m \boldsymbol{X}_{\boldsymbol{k}-i}^{\prime}}\right\} \\
& \cdot M\left\{e^{j 2 \pi f_{1}(q-m) \boldsymbol{X}_{\boldsymbol{k}-i}^{\prime \prime}}\right\} .
\end{aligned}
$$

The continuous random variable $\tau_{0}$ is uniformly distributed in the interval $T$

$M\left\{e^{j q 2 \pi f_{1} \tau_{0}}\right\}=\frac{1}{T} \int_{-T / 2}^{+T / 2} e^{j q 2 \pi f_{1} t} d t=\operatorname{sinc}\left(q f_{1} T\right)$.

The discrete random variable $k$ is uniformly distributed in the interval $2 h+1$

$$
\begin{aligned}
M\left\{e^{j q 2 \pi f_{1} \boldsymbol{k} T_{c}}\right\} & =\frac{1}{2 h+1} \sum_{k=-h}^{+h} e^{j q 2 \pi f_{1} \boldsymbol{k} T_{c}} \\
& =\frac{\operatorname{sinc}\left[q f_{1} T_{c}(2 h+1)\right]}{\operatorname{sinc}\left(q f_{1} T_{c}\right)} .
\end{aligned}
$$

By recalling that $\Phi\left(f T_{c}\right)$ is the characteristic function of the vector $\underline{\boldsymbol{X}}_{i}(6)$, we have

$$
M\left\{e^{j q 2 \pi f_{1} \boldsymbol{X}_{\boldsymbol{k}-i} T_{c}}\right\}=\int_{-\infty}^{+\infty} f(x) e^{j q 2 \pi f_{1} x T_{c}} d x=\Phi\left(q f_{1} T_{c}\right) .
$$

In the asynchronous or quasi-synchronous case, the asymptotic mean is derived by considering the limit, for $h$ tending to infinite, of (A3), and in the synchronous one the limit, for $T$ tending to infinite, of (A2) [6]; in both cases, it results in

$$
\stackrel{M}{\rightarrow}\left\{e^{j 2 \pi q_{1} \boldsymbol{k} T_{c}}\right\}=\underset{\rightarrow}{M}\left\{e^{j 2 \pi q f_{1} \tau_{0}}\right\}=\left\{\begin{array}{ll}
0, & \text { for } q \neq 0 \\
1, & \text { for } q=0
\end{array} .\right.
$$

Therefore, by recalling (5), the final expression of the asymptotic mean of $\hat{P}_{\boldsymbol{k}}$ given by (A1) becomes

$$
\underset{\rightarrow}{M}\left\{\hat{\boldsymbol{P}}_{\boldsymbol{k}}\right\}=\sum_{\substack{m=-\infty \\ m \neq 0}}^{+\infty} V_{m} I_{m}^{*}\left|\Phi_{1}\left(m f_{1} T_{c}\right)\right|^{2} \sum_{i=0}^{N-1} a_{i}
$$

where $\Phi_{1}\left(f T_{c}\right)$ is the common characteristic function of the vectors $\underline{\boldsymbol{X}}_{i}^{\prime}$ and $\underline{\boldsymbol{X}}_{i}^{\prime \prime}$.

To evaluate the asymptotic mean square error (13), we must first determine $M\left\{\hat{\boldsymbol{P}}_{\boldsymbol{k}}^{2}\right\}$. By introducing into (7) and (10) the random variables $\tau_{0}$ and $\boldsymbol{k}$, we can write

$$
=\sum_{q=-\infty}^{\left.\stackrel{M}{\rightarrow} \hat{\boldsymbol{P}}_{\boldsymbol{k}}^{2}\right\}} \sum_{q^{\prime}=-\infty}^{+\infty} \sum_{\substack{m=-\infty \\ m \neq q \\ m \neq 0}}^{+\infty} \sum_{\substack{m^{\prime}=-\infty \\ m^{\prime} \neq q^{\prime} \\ m^{\prime} \neq 0}}^{+\infty} V_{m} I_{q-m} V_{m^{\prime}} I_{q^{\prime}-m^{\prime}}
$$

$$
\begin{aligned}
& \sum_{i=0}^{N-1} \sum_{i^{\prime}=0}^{N-1} a_{i} a_{i^{\prime}} \\
& \text { - } \underset{\rightarrow}{M}\left\{e^{j 2 \pi f_{1}\left[m \boldsymbol{t}_{\boldsymbol{k}-i}^{\prime}+m^{\prime} \boldsymbol{t}_{\boldsymbol{k}-i}^{\prime}+(q-m) \boldsymbol{t}_{\boldsymbol{k}-i}^{\prime \prime}+\left(q^{\prime}-m^{\prime}\right) \boldsymbol{t}_{\boldsymbol{k}-i^{\prime}}^{\prime \prime}\right]}\right\} \\
& =\sum_{q=-\infty}^{+\infty} \sum_{q^{\prime}=-\infty}^{+\infty} \sum_{\substack{m=-\infty \\
m \neq q \\
m \neq 0}}^{+\infty} \sum_{\substack{m^{\prime}=-\infty \\
m^{\prime} \neq q^{\prime} \\
m^{\prime} \neq 0}}^{+\infty} V_{m} I_{q-m} V_{m^{\prime}} I_{q^{\prime}-m^{\prime}} \\
& \cdot \sum_{i=0}^{N-1} \sum_{i^{\prime}=0}^{N-1} a_{i} a_{i^{\prime}} \underset{\rightarrow}{\rightarrow}\left\{e^{j 2 \pi f_{1}\left(q+q^{\prime}\right) \pi_{0}}\right\} \underset{\rightarrow}{M}\left\{e^{j 2 \pi f_{1}\left(q+q^{\prime}\right) \boldsymbol{k} T_{c}}\right\} \\
& \cdot e^{-j 2 \pi f_{1}\left(q i+q^{\prime} i^{\prime}\right) T_{c}} \stackrel{M}{\longrightarrow}\left\{e^{j 2 \pi f_{1}\left(q \boldsymbol{X}_{\boldsymbol{k}-i}+q^{\prime} \boldsymbol{X}_{\boldsymbol{k}-i^{\prime}}\right) T_{c}}\right\} \\
& \cdot \underset{\rightarrow}{M}\left\{e^{j 2 \pi f_{1}\left[m \boldsymbol{t}_{\boldsymbol{k}-i}^{\prime}+m^{\prime} \boldsymbol{t}_{\boldsymbol{k}-i^{\prime}}^{\prime}+(q-m) \boldsymbol{X}_{\boldsymbol{k}-i}^{\prime \prime}+\left(q^{\prime}-m^{\prime}\right) \boldsymbol{X}_{\boldsymbol{k}-i^{\prime}}^{\prime \prime}\right] T_{c}}\right\} .
\end{aligned}
$$

By applying (A5), for $q+q^{\prime} \neq 0$, we obtain

$$
\underset{\rightarrow}{M}\left\{e^{j 2 \pi f_{1}\left(q+q^{\prime}\right) \boldsymbol{k} T_{c}}\right\}=\underset{\rightarrow}{M}\left\{e^{j 2 \pi f_{1}\left(q+q^{\prime}\right) \tau_{0}}\right\}=0 .
$$

For $q+q^{\prime}=0$ and $i=i^{\prime}$, recalling (5) we have

$$
\begin{aligned}
& e^{-j 2 \pi f_{1} q\left(i-i^{\prime}\right) T_{c}} \underset{M}{M}\left\{e^{j 2 \pi q f_{1} T_{c}\left(\boldsymbol{X}_{\boldsymbol{k}-i}-\boldsymbol{X}_{\boldsymbol{k}-i^{\prime}}\right)}\right\} \\
& \quad \cdot \stackrel{M}{\rightarrow}\left\{e^{j 2 \pi f_{1} T_{c}\left[m \boldsymbol{X}_{\boldsymbol{k}-i}^{\prime}+m^{\prime} \boldsymbol{X}_{\boldsymbol{k}-i^{\prime}}^{\prime}+(q-m) \boldsymbol{X}_{\boldsymbol{k}-i}^{\prime \prime}-\left(q+m^{\prime}\right) \boldsymbol{X}_{\boldsymbol{k}-i^{\prime}}^{\prime \prime}\right]}\right\} \\
&=\stackrel{M}{\rightarrow}\left\{e^{\left.j 2 \pi f_{1} T_{c}\left[\left(m+m^{\prime}\right) \boldsymbol{X}_{\boldsymbol{k}-i}^{\prime}-\boldsymbol{X}_{\boldsymbol{k}-i}^{\prime \prime}\right)\right]}\right\} \\
&=\left|\Phi_{1}\left(\left(m+m^{\prime}\right) f_{1} T_{c}\right)\right|^{2} .
\end{aligned}
$$

Finally, for $q+q^{\prime}=0$ and $i \neq i^{\prime}$, recalling (5) and (6) we have

$$
\begin{aligned}
e^{-j 2 \pi f_{1} q\left(i-i^{\prime}\right) T_{c}} \underset{\longrightarrow}{M}\left\{e^{j 2 \pi q f_{1} T_{c}\left(\boldsymbol{X}_{\boldsymbol{k}-i}-\boldsymbol{X}_{\boldsymbol{k}-i^{\prime}}\right)}\right\} & \stackrel{\longrightarrow}{\rightarrow}\left\{e^{j 2 \pi f_{1} T_{c}\left[m \boldsymbol{X}_{\boldsymbol{k}-i}^{\prime}+m^{\prime} \boldsymbol{X}_{\boldsymbol{k}-i^{\prime}}^{\prime}+(q-m) \boldsymbol{X}_{\boldsymbol{k}-i}^{\prime \prime}-\left(q+m^{\prime}\right) \boldsymbol{X}_{\boldsymbol{k}-i^{\prime}}^{\prime \prime}\right.}\right\} \\
= & e^{-j 2 \pi f_{1} q\left(i-i^{\prime}\right) T_{c}}\left|\Phi\left(q f_{1} T_{c}\right)\right|^{2} \Phi_{1}\left(m f_{1} T_{c}\right) \Phi_{1}\left(m^{\prime} f_{1} T_{c}\right) \\
& \cdot \Phi_{1}\left((q-m) f_{1} T_{c}\right) \Phi_{1}\left(\left(-q-m^{\prime}\right) f_{1} T_{c}\right) .
\end{aligned}
$$

We can summarize these results as follows:

$$
\begin{aligned}
& \underset{M}{M}\left\{e^{j 2 \pi f_{1}\left[m t_{k-i}^{\prime}+m^{\prime} t_{k-i^{\prime}}^{\prime}+(q-m) t_{k-i}^{\prime \prime}+\left(q^{\prime}-m^{\prime}\right) t_{k-i^{\prime}}^{\prime}\right]}\right. \\
& \quad= \begin{cases}0, & \text { for } q \neq-q^{\prime} \\
(\mathrm{A} 9), & \text { for } q=-q^{\prime} \text { and } i=i^{\prime} \\
(\mathrm{A} 10), & \text { for } q=-q^{\prime} \text { and } i \neq i^{\prime}\end{cases}
\end{aligned}
$$

By recalling these results and the equality

$$
\sum_{i=0}^{N-1} \sum_{i^{\prime}=0}^{N-1}(\cdot)=\sum_{i=0}^{N-1} \sum_{\substack{i^{\prime}=0 \\ i=i^{\prime}}}^{N-1}(\cdot)+\sum_{i=0}^{N-1} \sum_{\substack{i^{\prime}=0 \\ i \neq i^{\prime}}}^{N-1}(\cdot)
$$




$$
\begin{aligned}
M\left\{\hat{\boldsymbol{P}}_{\boldsymbol{k}}^{2}\right\}= & \sum_{q=-\infty}^{+\infty} \sum_{\substack{m=-\infty \\
m \neq q \\
m \neq 0}}^{+\infty} \sum_{\substack{m^{\prime}=-\infty \\
m^{\prime} \neq q^{\prime} \\
m^{\prime} \neq 0}}^{+\infty} V_{m} I_{q-m} V_{m^{\prime}} I_{-q-m^{\prime}}\left|\Phi_{1}\left(\left(m+m^{\prime}\right) f_{1} T_{c}\right)\right|^{2} \sum_{i=0}^{N-1} a_{i}^{2} \\
& +\sum_{q=-\infty}^{+\infty} \sum_{\substack{m=-\infty \\
m \neq q \\
m \neq 0}}^{+\infty} \sum_{\substack{m^{\prime}=-\infty \\
m^{\prime} \neq-q \\
m^{\prime} \neq 0}}^{+\infty} V_{m} \Phi_{1}\left(m f_{1} T_{c}\right) I_{q-m} \Phi_{1} \\
& \cdot\left((q-m) f_{1} T_{c}\right) V_{m^{\prime}} \Phi_{1}\left(m^{\prime} f_{1} T_{c}\right) I_{-q-m^{\prime}} \Phi_{1}\left(\left(-q-m^{\prime}\right) f_{1} T_{c}\right)\left|\Phi\left(q f_{1} T_{c}\right)\right|^{2}\left[\left|H\left(q f_{1} T_{c}\right)\right|^{2}-\sum_{i=0}^{N-1} a_{i}^{2}\right] \\
& \left.\sum_{q=-\infty}^{+\infty} \sum_{\substack{m=-\infty \\
m \neq q \\
m \neq 0}}^{+\infty} \sum_{\substack{m^{\prime}=-\infty \\
m^{\prime} \neq-q \\
m^{\prime} \neq 0}}^{+\infty} V_{m} I_{q-m} V_{m^{\prime}} I_{-q-m^{\prime}} \mid \Phi_{1}\left(m+m^{\prime}\right) f_{1} T_{c}\right)\left.\right|^{2} \sum_{i=0}^{N-1} a_{i}^{2} \\
& +\sum_{q=-\infty}^{+\infty}\left|\sum_{\substack{m=-\infty \\
m \neq q \\
m \neq 0 \\
m \neq 0}}^{+\infty} V_{m} \Phi_{1}\left(m f_{1} T_{c}\right) I_{q-m} \Phi_{1}\left((q-m) f_{1} T_{c}\right)\right|^{2}\left|\Phi\left(q f_{1} T_{c}\right)\right|^{2}\left[\left|H\left(q f_{1} T_{c}\right)\right|^{2}-\sum_{i=0}^{N-1} a_{i}^{2}\right]
\end{aligned}
$$

(A7) becomes (A13), shown at the top of the page, where

$$
H(f)=\sum_{i=0}^{N-1} a_{i} e^{-j 2 \pi f i T_{c}}
$$

is the frequency response of the digital filter.

\section{REFERENCES}

[1] T. M. Sunders, D. R. Flach, C. Hagwood, and G. L. Yang, "The effects of timing jitter in sampling systems," IEEE Trans. Instrum. Meas., vol. 39, pp. 80-85, Feb. 1990.

[2] S. S. Award and M. F. Wagdy, "More on jitter effects on sinewave measurements," IEEE Trans. Instrum. Meas., vol. 40, pp. 549-552, June 1991.

[3] M. Sun, D. L. Bonaddio, J. Mi, and R. J. Sclabassi, "An analysis of the effects of jitter in data acquisition on synchronous averaging," IEEE Trans. Syst., Man, Cybern., vol. 21, pp. 456-463, Mar./Apr. 1991.

[4] D. Mirri, G. Iuculano, F. Filicori, and G. Pasini, "Performance function for time-jittered equispaced sampling wattmeters," IEEE Trans. Instrum. Meas., vol. 44, pp. 671-674, June 1995.

[5] F. Filicori, G. Iuculano, A. Menchetti, D. Mirri, and M. Catelani, "New performance function for the comparison of different sampling strategies in nonlinear conversion instruments," in Proc. IEEE Instrumentation Measurement Technol. Conf., Washington, DC, Apr. 1989, pp. 307-311.

[6] G. Iuculano, D. Mirri, F. Filicori, A. Menchetti, and M. Catelani, "A criterion for the analysis of synchronous and asynchronous sampling instruments based on non linear processing," Proc. Inst. Elect. Eng., vol. 139, pt. A, no. 4, pp. 141-152, July 1992.

[7] D. Mirri, F. Filicori, G. Iuculano, A. Menchetti, and M. Catelani, "Accuracy evaluation of digital wattmeters based on different sampling strategies," in IMEKO TC7 Symp. on Knowledge Based Measurements, Karlsruhe, Germany, Sept. 1990, pp. 117-124.

[8] A. Papoulis, The Fourier Integral and its Applications. New York: McGraw-Hill, 1962.

[9] F. J. J. Clarke and J. R. Stockton, "Principle and theory of wattmeters operating on the basis of regularly spaced sample pairs," J. Phys. E, pp. 645-652, 1982.

[10] F. Filicori, G. Iuculano, A. Menchetti, and D. Mirri, "A random asynchronous sampling strategy for measurement instrument based on nonlinear signal conversion," Proc. Inst. Elect. Eng., vol. 136, pt. A, no. 3, pp. 141-150, May 1989.

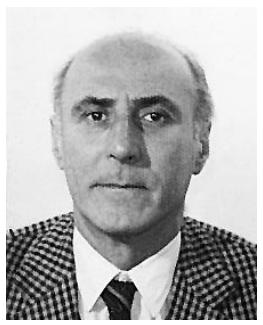

Domenico Mirri was born in Italy in 1936. He graduated with a degree in electronic engineering from the University of Bologna, Bologna, Italy.

At present, he is Associate Professor of Electronic Measurement at the University of Bologna. His current research interests are in the areas of digital measurement instruments, device metrological characterization, and biomedical measurements.

Gaetano Iuculano received the degree in electronic engineering.

At present, he is Full Professor of Electrical Measurements and Metrology at the University of Florence, Florence, Italy. His current research interests are calibration applications, reliability analysis and life testing for electronic devices and systems, statistical analysis, and digital measurement instruments.

Gaetano Pasini was born in Italy in 1964. He graduated with a degree in electronic engineering from the University of Bologna, Bologna, Italy.

$\mathrm{He}$ is presently a Researcher in Electrical Measurement at the University of Bologna. His research interests mainly involve digital signal processing and power measurements.

Fabio Filicori was born in Italy in 1949. He graduated with a degree in electronic engineering from the University of Bologna, Bologna, Italy, in 1964.

At present, he is Full Professor at the University of Bologna. His current research interests are in the areas of nonlinear circuit analysis and design, electronic devices modeling, digital measurement instruments, and power electronics. 